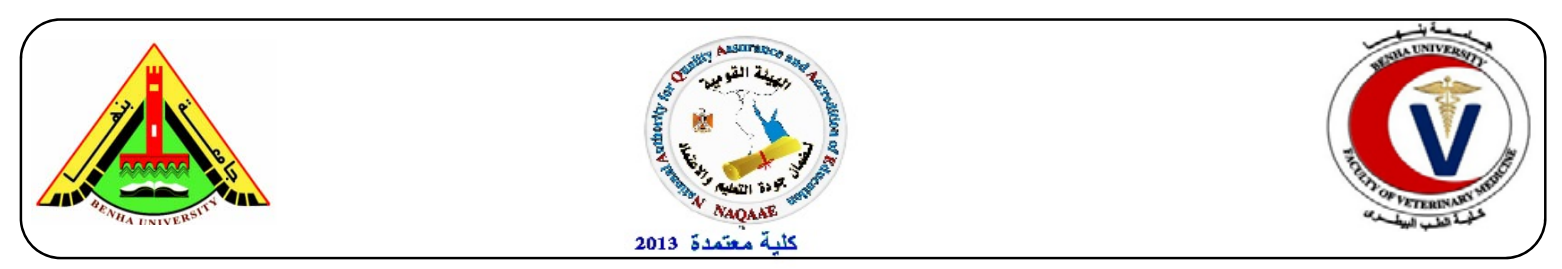

\title{
Detection and pathotyping of a recent Newcastle disease virus outbreak in Egypt
}

Gabr. F. El-Bagoury ${ }^{1}$, Ehab. M. El-Nahas ${ }^{1}$, Mahmoud. M. Abd-El-Monem², Mohamed. A. AboElkhair ${ }^{2}$

${ }^{1}$ Department of virology, Faculty of Veterinary Medicine, Benha University. ${ }^{2}$ Veterinary Serum and Vaccine Research Institute, Abbassia, Cairo.

\section{A B S T R A C T}

This study was conducted to detect and pathotype Newcastle disease virus (NDV) isolates from a recent outbreaks affecting poultry farms in Egypt between 2013 and 2014. Trachea, liver and brains were collected from clinically infected NDV three different vaccinated broiler farms in Sharqia Province. The reverse transcription polymerase chain reaction (RT-PCR) using fusion (F) gene specific primers were more sensitive than haemagglutination (HA) in the detection of NDV in collected samples. Inoculation of all the collected samples in 10-day-old embryonated chicken specific-pathogen-free eggs resulted in isolation of haemagglutinating agents in 18 samples. These haemagglutinating agents were confirmed as NDV by haemagglutination inhibition (HI) test. The intracerebral pathogenicity index (ICPI) revealed that all isolates have a range $>1.5$ index which congruent to velogenic type. These results confirmed that the circulating NDV strains are virulent for chickens and vaccination failures occur.

Keywords: NDV, Chicken, ICPI, RT-PCR

(BVMJ-29(2): 297-302, 2015)

\section{INTRODUCTION}

$\mathrm{N}$ ewcastle disease virus (NDV), also referred to as avian paramyxovirus type 1 (APMV-1), causes Newcastle disease (ND) in more than 200 bird species (OIE, 2011). NDV is a member of the genus Avulavirus, family Paramyxoviridae. Paramyxoviruses are single-stranded RNA viruses with a genome size of approximately $15 \mathrm{~kb}$ with a genomic arrangement of six genes coding for six polypeptides (Lamb and Parks, 2002). Although NDV infects a variety of bird species, it is most virulent in chickens, and in spite of 60 years of concerted vaccination strategies, ND is still a very serious problem of the poultry industry worldwide (Czegledi et al., 2006). Signs associated with NDV infection can range from very mild respiratory problems and mild enteric disease to extremely high mortality rates in flocks (Aldous et al., 2003). The broad spectrum of signs evident in NDV-infected birds derives from five different pathotypes of differing severity. The pathotypes include asymptomatic enteric and lentogenic variants, which are commonly employed as live vaccines, mesogenic viruses, and viscerotropic and neurotropic velogenic variants, of which the last two are extremely pathogenic (Fratnik Steyer et al., 2010).

In Egypt, NDV outbreaks are occurring frequently, and the rapid and accurate diagnosis of ND are essential. Therefore, the present study was conducted to rapidly detect and pathotype NDV isolated from the recent outbreak in sharqia province, Egypt.

\section{MATERIAL AND METHODS}

\subsection{Samples Collection}

Within two years of this study (January 2013 to December 2014), forty ND- 
suspected field samples (trachea, liver and brain tissues) were obtained from chickens flocks suspected to have ND in three broiler farms of Sharqia Province (Table 1 and 2). Most sacrificed chickens had diarrhea, also showed nervous symptoms and respiratory difficulties. These samples were labeled and transported immediately on ice to the laboratory to be either processed immediately or stored at $-80^{\circ} \mathrm{C}$ until processed and inoculated into embryonated chicken egg (ECE).

Table 1. Historical data of the ND outbreaks among vaccinated broiler chicken flock in Sharqia province, Egypt during 2013-2014

\begin{tabular}{lcccc}
\hline farm & $\begin{array}{c}\text { Sample } \\
\text { No. }\end{array}$ & age & $\begin{array}{c}\text { Flock } \\
\text { capacity }\end{array}$ & $\begin{array}{c}\text { Mortality } \\
\%\end{array}$ \\
\hline I & 20 & 26 & 2500 & 80 \\
II & 10 & 18 & 2000 & 70 \\
III & 10 & 30 & 1500 & 75 \\
\hline
\end{tabular}

Table 2: Types and numbers of the collected samples

\begin{tabular}{lccc}
\hline farm & trachea & liver & brain \\
\hline I & 7 & 7 & 6 \\
II & 4 & 4 & 2 \\
III & 4 & 4 & 2 \\
total & 15 & 15 & 10 \\
\hline
\end{tabular}

\subsection{Virus isolation in ECE}

A $10 \%$ tissue suspension was prepared from the collected organs as previously described (Terregino and Capua, 2009). The suspension was left at $4^{\circ} \mathrm{C}$ overnight and clarified by centrifuging at $2000 \mathrm{rpm}$ for 10 min at room temperature. A $0.2 \mathrm{ml}$ of the supernatant from different samples (trachea, liver and brain) was inoculated in duplicate into 9-11 day-old ECE via allantoic cavity. The inoculated eggs were incubated at $37^{\circ} \mathrm{C}$ for 5-7 days with daily observing the embryo viability. Deaths occurred during the first $24 \mathrm{hr}$ of incubation was considered non-specific death. All the embryos that died after $24 \mathrm{hr}$ or survived till the end of incubation period were chilled in refrigerator $\left(4^{\circ} \mathrm{C}\right)$ overnight. The allantoic fluid (AF) was harvested, divided into aliquots and stored in sterile screw-capped vials at $-80^{\circ} \mathrm{C}$ till further use.

\subsection{Serological Detection and Identification of NDV}

The presence of NDV in the collected samples and AF were determined by slide HA test, micro-titer plate HA and HI tests following the standard procedure (Grimes, 2002). Briefly, the HA test was performed using chicken red blood cells (RBCs) in 96well V-bottom mixed with an equal volume of a $1 \%(\mathrm{v} / \mathrm{v})$ RBCs in a V bottomed 96well micro-titer plate. The plate was then incubated for $30 \mathrm{~min}$ at RT. The titers were expressed as reciprocals of the highest dilution of virus that demonstrated RBCs agglutination. The HA negative AF was passaged twice in ECE before recorded as NDV negative sample. For HI test, serial twofold anti-NDV serum dilutions were made in PBS; 4 HA units of tested AF was added to each dilution and incubated at RT for $30 \mathrm{~min}$. after that an equal volume of $1 \%$ chicken RBCS in PBS was added. The HI endpoint was determined as the last dilution with inhibition of HA activity.

\subsection{Molecular Detection of NDV in suspected samples}

Viral RNAs were extracted from the samples of trachea, liver and brain suspensions with QIA amp Viral RNA mini kits (Qiagen, Inc., Valencia, CA) according to the manufacture instructions. The RTPCR was carried out using oligonucleotide primers for NDV-F gene including, sense primer 5'- GGA GGA TGT TGG CAG CATT-3'and antisense primer 5'- GTC AAC ATA TAC ACC TCATC-3'with final product $318 \mathrm{bp}$. The RT-PCR was performed according to the method described by (Gopinath et al., 2011) in 50 $\mu l$ of a reaction mixture containing a final concentration of $10 \mathrm{mM}$ Tris ( $\mathrm{pH}$ 9.0), 50 $\mathrm{mM} \mathrm{KCl}, 0.01 \%$ gelatin, $1.9 \mathrm{mM} \mathrm{MgCl}$, $5 \%$ (wt/vol) glycerol, $0.2 \mathrm{mM}$ deoxynucleotide triphosphate, $0.1 \mathrm{M}$ of 
each primer listed in Table 2, $10 \mathrm{U}$ of Taq polymerase per $\mu \mathrm{l}$ and $5 \mu \mathrm{l}$ of extracted NDV-RNA. The PCR mixtures were subjected to 38 repeated cycles of amplification in a DNA thermal cycler. The cycling conditions was as follows: denaturation $95^{\circ} \mathrm{C}$ for $1 \mathrm{~min}$, primer annealing at $60^{\circ} \mathrm{C}$ for $1 \mathrm{~min}$ and extension at $72^{\circ} \mathrm{C}$ for $1 \mathrm{~min}$. Negative and positive control reactions was included. The PCR products were detected by electrophoresis through a $1.5 \%$ agarose gel and visualization under UV light after ethidium bromide staining.

\subsection{ICPI for the NDV Isolates}

It was performed to evaluate the pathogenicity of NDV isolates. The test was applied on 180 one-day old chicks according to international OIE standards (2012) obtained from unvaccinated flocks, also random serum samples from these chicks were confirmed by $\mathrm{HI}$ as NDV negative. Fresh AF obtained after passaging the NDV isolates in ECE with a HA titer > $2^{4}(>1 / 16)$ was diluted $1 / 10$ in sterile PBS with no additives, such as antibiotics. Then $0.05 \mathrm{ml}$ of the diluted virus was injected intra-cerebral (I/C) in the chick (10 chicks / sample), as well as one group (10 chicks) was injected with $0.05 \mathrm{ml}$ PBS as control. The birds were observed for the clinical symptoms every 24h/ 8 days. At each observation, the birds were scored: normal $(0)$, sick (1) and dead (2). The quotient derived from the sum of scores and the numbers of observations represent the ICPI. An ICPI above 1.5 characterizes as a velogenic strain, $0.5-1.5$ as a mesogenic strain; while an ICPI below 0.5 indicates a lentogenic strain.

\section{RESULTS}

\subsection{Detection of NDV in collected samples}

The HA antigen were detected in 18 out of 40 samples including, seven tracheal, seven liver and four brain samples. These haemagglutinating agents were confirmed as NDV by haemagglutination inhibition (HI) test. The RT-PCRs for NDV were positive for 25 out of 40 samples including nine tracheal, nine liver and seven brain samples (table 3). The amplified PCR products of $318 \mathrm{bp}$ were analyzed by agarose gel electrophoresis and visualized by ultraviolet rays with ethidium bromide (Fig. 1).

\subsection{Characterization of NDV using Haemagglutination (HA)}

Totally the HA titer was relatively increased post isolation on ECE after 3 successive passages with 2 to $3 \log$ for HA antigen pre isolation. A higher HA titers were observed with the brain suspensions followed by tracheal and liver suspension indicates the neurotropic affinity of these isolates (Table 4).

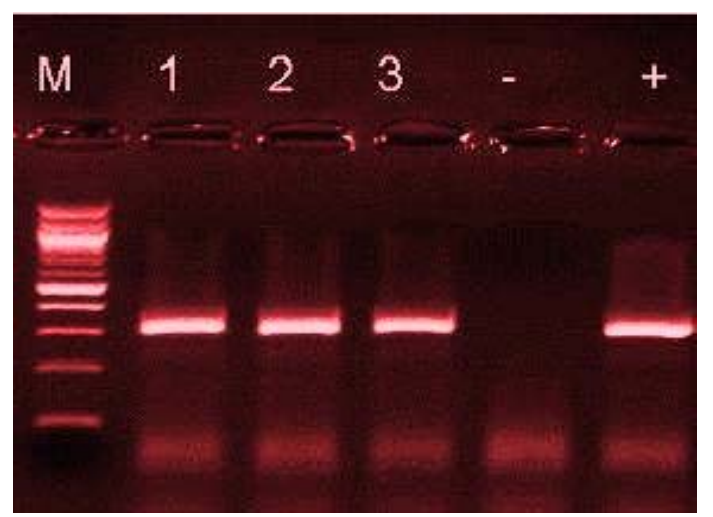

Fig. 1: RT-PCR results of trachea, liver and brain samples from infected broiler chickens with primer specific to fusion gene of NDV. M=100bp DNA ladder. Lane 1: tracheal sample. Lane 2: liver sample. Lane $3=$ brain sample. Lanes $-=$ negative control. Lanes $+=$ positive control Samples were positive with an expected size (318bp) amplicon

\subsection{Pathotyping of NDV isolates in chicken using ICPI}

The 18 isolate of NDV that were detected by both HA and RT-PCR have an ICPI value $>1.5$ (table. 5) indicate its velogenic nature. 
Table 3: Comparison of HA and RT-PCR for identification of NDV in the collected samples

\begin{tabular}{|c|c|c|c|c|c|c|c|c|}
\hline \multirow{2}{*}{ farm } & \multicolumn{3}{|c|}{ HA } & \multirow{2}{*}{ total } & \multicolumn{3}{|c|}{ RT-PCR } & \multirow{2}{*}{ total } \\
\hline & trachea & liver & brain & & trachea & liver & brain & \\
\hline I & 3 & 3 & 2 & 8 & 4 & 4 & 3 & 11 \\
\hline II & 3 & 2 & 1 & 6 & 3 & 3 & 2 & 8 \\
\hline III & 1 & 2 & 1 & 4 & 2 & 2 & 2 & 6 \\
\hline total & 7 & 7 & 4 & $18(100)$ & 9 & 9 & 7 & $25(100)$ \\
\hline
\end{tabular}

Table 4: the distribution of HA antigen of NDV isolates obtained from the collected samples in Sharqia province, Egypt

\begin{tabular}{lcccccccc}
\hline \multirow{2}{*}{ farm } & \multicolumn{3}{c}{ HA titer pre isolation } & & \multicolumn{3}{c}{ HA titer post isolation } & \multirow{2}{*}{ total } \\
\cline { 2 - 3 } & $1 / 64$ & $1 / 128$ & $1 / 256$ & & $1 / 256$ & $1 / 512$ & $1 / 1024$ & \\
\hline trachea & 3 & 3 & 1 & 7 & 3 & 4 & 0 & 7 \\
liver & 3 & 4 & 0 & 7 & 3 & 4 & 0 & 7 \\
brain & 0 & 1 & 3 & 4 & 0 & 1 & 3 & 4 \\
total & 6 & 8 & 4 & $18(100)$ & 6 & 9 & 3 & $18(100)$ \\
\hline
\end{tabular}

Table 5: The ICPI values and pathotypes of NDV strains used in this study

\begin{tabular}{|c|c|c|c|c|c|c|}
\hline \multirow{2}{*}{ ICPI } & \multicolumn{3}{|c|}{ Tissue sample } & \multirow{2}{*}{$\begin{array}{c}\text { NDV } \\
\text { pathotype }\end{array}$} & \multirow{2}{*}{$\begin{array}{l}\text { Virulence in } \\
\text { chiken }\end{array}$} & \multirow{2}{*}{ percen } \\
\hline & trachea & liver & brain & & & \\
\hline$<0.5$ & 0 & 0 & 0 & - & - & 0 \\
\hline $0.5-1.5$ & 0 & 0 & 0 & - & - & 0 \\
\hline$>1.5$ & 7 & 7 & 4 & velogenic & high & 100 \\
\hline
\end{tabular}

\section{DISCUSSION}

Despite the intensive vaccination regimes carried out in Egypt to combat NDV, many outbreaks of NDV still reported and the disease causes serious economic losses in the poultry industry.

This is may be due to many reasons including the random use of intensive vaccines, frequent mutations of the NDV, and emerging of new pathotypes of NDV (Pchelkina et al., 2013).

So, the isolation and pathotyping of the NDVs from recent outbreaks among chickens is acritical for the control of ND and vaccination evaluation.

In the last 50 years there has been a major genetic change in the strains of NDV that have been identified in poultry, although they still remain as a single serotype (Miller et al., 2007). 
Many techniques have been developed to diagnose NDV. Comparing the sensitivity of some conventional techniques such as (HA, isolation via ECE, and $\mathrm{HI}$ ) to some new techniques such as (PCR, Real Time PCR and ELISA) for the detection of NDV. Our results are showing that RT-PCR is more superior to HA in the detection of NDV in different samples (Table 3, Figure 1). Our results are consistent with other studies which found that PCR is more sensitive than HA in the early detection of NDV (Mohammed et al., 2013).

Pathotyping of NDV was mainly dependant of the Pathogenicity index either the intracerebral (ICPI) or intravenous pathogenicity index (IVPI) or the mean death time (MDT) (Mishra et al., 2001). The ICPI of the identified isolates revealed its virulence to chickens producing characteristic clinical and PM features of NDV in infected chicks (Fig. 2) with a high incidence of the velogenic NDV isolates $(100 \%)$ in Sharqia province (Table 5). The NDV infection even in a well-vaccinated flock can occur because some of the birds will have had a poor vaccine response and will be susceptible to infection. This attributed to ND vaccines do not protect vaccinates from infection and viral shedding. Also, parental immunity contributed to vaccination inefficiency in young chicks (Kapczynski and King, 2005). In conclusion, NDV isolates circulating among chickens are virulent and associated with outbreaks in commercial poultry farms in Sharqia province. Molecular based techniques are more superior to the conventional ones in the early rapid and sensitive detection of NDV in the affected chicken flocks. Establishing molecular analysis to this strain will open several avenues toward diagnosis and local vaccine preparation.

\section{REFERENCES}


Lamb RA, Parks GD, 2002. Paramyxovirinae: the viruses and their replication. In: Knipe DM, Howley PM (eds) Fields virology, vol 1.

Miller, P. J., King, D. J. Afonso, C. L. Suarez, D. L. 2007. Antigenic differences among Newcastle disease virus strains of different genotypes used in vaccine formulation affect viral shedding after a virulent challenge. Vaccine, 25(41):72387246.

Mishra, S. Kataria, J.M., Sah, R.L., Verma, K.C., Mishra, J.P. 2001. Studies on the pathogenicity of Newcastle disease virus isolates in guinea fowl. Trop Anim Health Prod., 33(4):31320.

Mohammed, M. H., Zahid, A.H., Kadhim, L.I., Hasoon, M.F. 2013. Conventional and Molecular Detection of Newcastle Disease and Infectious Bursal Disease in Chickens J World's Poult Res. 3(1):05-12.

OIE, 2011. Newcastle Disease, In: Manual of diagnostic tests and vaccines for.
Lippincott Williams \& Wilkins Inc, Philadelphia, Chapter 41, pp 14491496.

OIE, 2012. Newcastle disease, Chapter 2. 3.14. OlE Manual of Standards for Diagnostic Tests and Vaccines, NB: Version adopted by the World Assembly of Delegates of the OIE in May.

Pchelkina, I.P., Manin, T.B., Kolosov, S.N., Starov, S.K., Andriyasov, A.V., Chvala I.A, 2013. Characteristics of pigeon paramyxovirus serotype-1 isolates (PPMV-1) from the Russian Federation from 2001 to 2009. Avian Dis. 57(1):2-7.

Terregino, C., Capua, I. 2009. Conventional diagnosis of Newcastle disease virus infection, In: Avian influenza and Newcastle disease, afield and laboratory manual. I. Capua and D.J. Alexander, ed., Springer Milan, Milan, Italy, pp. 123-125. 